\title{
Food choices and preferences of schoolchildren
}

\author{
BY JANE THOMAS \\ Department of Food and Nutritional Sciences, King's College London, Campden Hill Road, \\ London W8 $7 A H$
}

While other speakers have considered what children are eating and the nutritional implications, this paper will take a closer look at why children eat in the way that they do.

Clearly food habits are shaped by many factors which limit the availability and acceptability of potential foodstuffs. But within this framework, humans also form strong, stable likes and dislikes for foods. Even among children as young as 4 years of age, Birch (1979a) has shown that preference rankings made at one time, are good predictors of rankings made up to $51 \mathrm{~d}$ later. Furthermore preference rankings made by pre-school age children are highly correlated with consumption (Birch, 1979b).

How do children acquire such preferences and food patterns? Birch (1987) has proposed three non-independent forms of transmission.

1. Infants are born with genetically pre-programmed propensities for behaviour.

- This would suggest that food patterns result from innate mechanisms.

2. Similar food acceptance may result from similar exposure to and experience with food across the generations.

- The similarity of exposure is due to social constraints on food experience. Such constraints limit what foods children are exposed to and in what context exposure occurs.

3. Transmission of behaviour can result from direct social interaction among individuals of a social group.

- In humans, this form of transmission frequently involves an individual who acts as an agent of socialization in the culture, transmitting cultural rules to the child. (Interestingly, Galef (1986) appears to have demonstrated that social interaction can modify learned aversions, sodium appetite and palatability in rats.)

The relative strength of these factors is of considerable importance in trying to understand how people make food choices, particularly for those concerned with changing eating habits. However, there seems little doubt that these factors interact and on the basis of the information available it seems reasonable to concur with Pliner \& Pelchat (1986) that, 'The human omnivore is an animal that basically has to learn what to eat, with some universal genetic predispositions, but with little influence of genetic factors on individual differences in food choice and attitudes'. The sequence in which this learning occurs is sometimes described as a 'career' and naturally the relative importance of factors which impinge on the learning process vary over time. This paper will consider how children's food preferences appear to develop and comment on some of the implications for nutrition education.

\section{THE INFANT: INNATE PREDISPOSITIONS AND THE EFFECTS OF LEARNING}

The question of whether or not humans have an innate ability to select a balanced diet (gustatory sensibility) is a controversial one. While the evidence of preferences based on 
needs for individual nutrients is scanty in humans, there is considerably more experimental evidence which suggests that adjustments to eating behaviour do occur in response to energy intake, particularly amongst infants (Fomon, 1974).

An innate preference in humans for the sweet taste has been widely documented. The human newborn prefers sugar solutions to water (Crook, 1978; Desor et al. 1973) and even to formula (Desor et al. 1977), while older children have been shown to have a continuing preference for the sweet taste (Desor et al. 1975).

However, even this inborn sweet preference may be modified in childhood. Experience with sweet foods, especially early in life, has been proposed as one factor that may affect the formation and modification of food preferences (Beauchamp \& Moran, 1982). Birch et al. (1980) have pointed out that experience, in terms of the context in which foods are presented, is usually positive for sweet foods. The positive associations created by giving a child sweet foods as a reward and on special occasions may contribute to strengthening the preference for a sweet taste. Controlled studies (Birch et al. 1980; Birch, 1981) showed that the presentation of foods (both sweet and non-sweet) in a positive context resulted in at least short-term preferences for these foods so that even in the instance where there is a clear predisposition, it can be subsequently modified by learning.

\section{THE YOUNG CHILD: THE ROLE OF CARETAKERS}

For the young child, adult caretakers severely limit experience with food which can have effects on the preferences a child expresses. Since 'neophobia', a fear of new things, is a basic human characteristic which extends to new foods, it is perhaps not surprising that a number of authors have demonstrated that young children like best the foods to which they have been exposed most often, or conversely 'I've never tasted it - I don'l like it!'.

Clearly religious and economic factors will affect the foods to which a child is exposed and in most cultures certain foods are seen to be inappropriate for children. Jerome (1977) notes that young children are rarely given lobster, caviar or coffee. While Rozin \& Schiller (1980) point out that even in countries where hot, spicy foods play a central part in the diet such foods are not given to young children.

It is not always easy to separate the extent to which the influence of an adult is operating through the role of 'gatekeeper' or directly through the third suggested route of social interaction.

Early work by Escalona (1945) showed that young children would change their preference for orange or tomato juice depending on those of their caretaker, even when both were equally available, while Ritchey \& Olsen (1983) have shown that frequency of sweet-eating among pre-school children was related to the parents' own frequency of eating sweets, parents giving sweets in a positive context and the amount of television that children watched. The literature on the extent of similarity between parental food preferences and those of young children has identified a number of interesting points. As modelling theory would predict, the preferences of female children more closely resemble those of the mother and those of male children resemble those of the father. Mother-child similarity in eating habits is greater than could be accounted for by membership of the same subcultural group. However, the similarity between the preferences of children and their father was no greater than that of unrelated child-adult pairs from the same subcultural group (Birch, 1987). 
However, Macarthy (1935), Pliner (1983) and others have found the greatest degree of similarity in food preferences between siblings. The percentage of foods disliked or rejected in common with siblings (76) far exceeded the percentage of foods disliked or rejected in common with parents (24), among her pre-school subjects. This may be due to the greater general similarity between a young child and its sibling; this similarity may make the sibling a more potent model initiating imitation, while in addition the siblings share a more similar pattern of exposure to foods with each other, than they do with their parents.

A further way in which parents may affect their children's eating habits and preferences is through direct attempts to bring about change.

Casey \& Rozin (1989) looked at what methods parents believed could be used to change children's eating habits and compared these with their actual practices. The methods which parents believed would be most effective for creating food "likes" included, showing the children that the adults like the food; involving the child in its preparation; telling the child that siblings liked it and creating a positive social context when offering the food. When parents were asked what methods they actually used, these were, showing that adults liked the food: letting the child help to prepare; telling the child it was good for them and rewarding them for eating the particular food. It is interesting to note that the parents favoured giving a reward for eating the food rather than using the food that they wished to encourage as the reward, which experimental work (Birch, 1987) suggests is more effective; e.g. if the child is to be encouraged to eat green beans, it is more effective to say 'if you hurry up and come straight to lunch you can have some green beans' than to say 'eat up your green beans and then you can have some ice cream'. The positive value is likely to be attached to the reward, in the latter case it becomes attached to the ice cream rather than the green beans.

\section{GOING TO SCHOOL: A WIDER WORLD}

This brings an increase in contact with peers in eating situations and the effects of this social interaction have been demonstrated by Birch (1980). A target child with a strong preference for a particular vegetable $A$, was placed at a lunch table with three or four children who preferred another vegetable, B. On the first day, vegetables A and B were offered and the minority child allowed to choose first. On the three successive days this child chose last. Over this period the three or four other children increasingly chose the non-preferred vegetable and afterwards reported an increased preference rating for this vegetable, while no changes occurred in the control group.

Greater contact with peers may contribute to a broadening dichotomy, identified by James (1981) between adults' and children's perceptions of eating and foods. She illustrated her case with special reference to 'Kets', a word used in the North-East of England to denote 'rubbish', which children also used to describe popular cheap confectionery. Kets differ from ordinary sweets in several ways: names, colours, sensations they produce as well as the time and manner of consumption. They have distinctive names: jelly babies, gobstoppers. Often they have lurid colours and produce exciting taste sensations, fizzy sherbet or acid flavours. These differ from 'sweets' which are considered to be more like the real food which is eaten at mealtimes. Those are usually wrapped and ingredients may be listed on the label. In contrast 'kets' are usually unwrapped; they are sold loose and children may rummage around to make their 


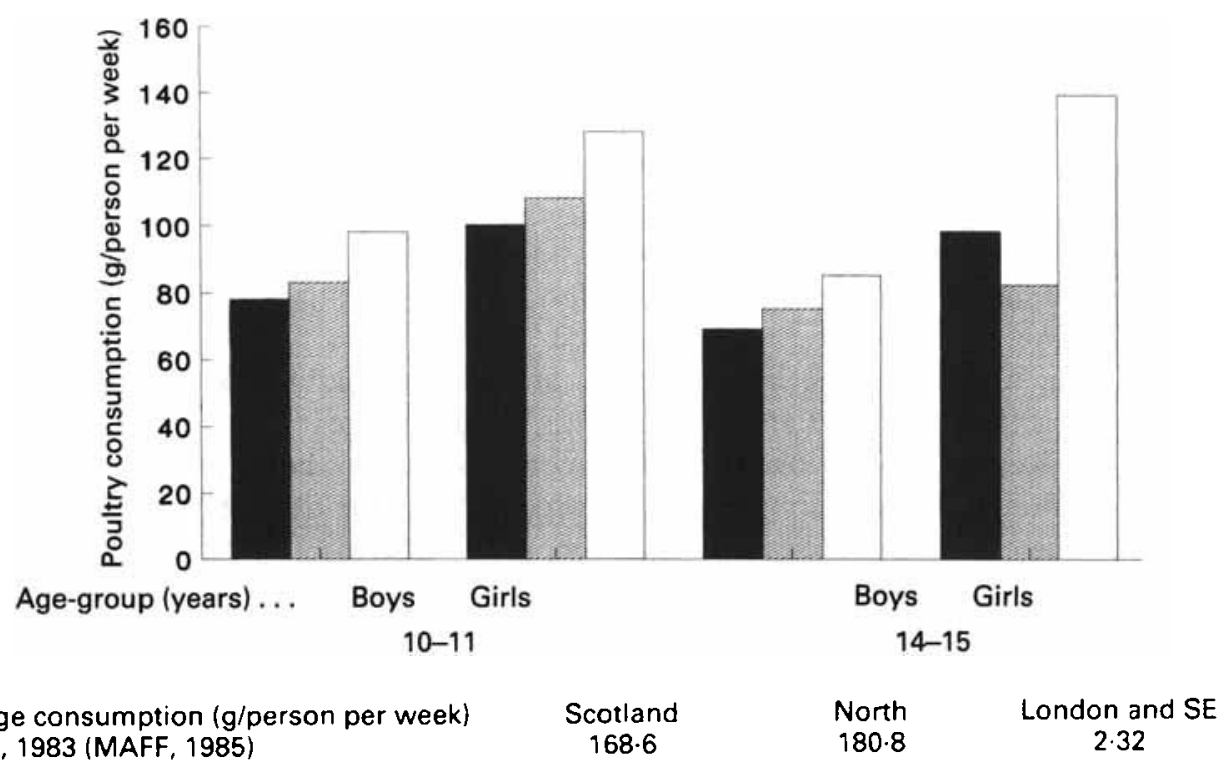

Average consumption (g/person per week) NFS, 1983 (MAFF, 1985)

Scotland
$168 \cdot 6$

$180 \cdot 8$

$2 \cdot 32$

Fig. 1. Poultry consumption by age and region (DHSS, 1989). Scotland: $\mathbb{Z}$ North: [ London and SE.

selection; they are shared freely with friends and exchanged; children often take them in and out of their mouths. James (1981) suggests that by eating 'kets' children are literally chewing up the adult order of things. Prestige can be established by different criteria from those which adults apply; children display the virtuosity of their knowledge about 'kets', insisting on using the correct names and knowing the detailed characteristics of each variety.

Rousseau (1984) explored this further amongst primary schoolchildren in Edinburgh. She compared the foods that were eaten with peers to those which were eaten with adults. Children's eating, which took place alone or with peers was more likely to take place between meals and in unstructured settings without utensils. Children's foods were characterized by differences in terms of: taste, strong or tart; texture, hard, crispy; temperature, room temperature or frozen; shape, animal, monster etc.; size, bite-sized; visual aspects, vivid colours, interesting containers. Ideally these foods should also be highly portable and easy to share.

In a further experiment, Rousseau (1984) presented popular foods, like Smarties and Monster Munch in dull packaging and other items like walnuts, dates and pure orange juice in more attractive forms and was able to produce significant changes in appeal. These differences in food ideology can clearly have implications for food choice and nutrient intakes. Prattala (1988) looking at sociodemographic differences in fat and sugar consumption among Finnish adolescents, found socio-economic variables strongly linked with the foods that adolescents ate at home, whereas those foods bought outside the home were independent of family background, showing a distinction in adolescents' use of foods.

Turning to diets of British schoolchildren (Department of Health and Social Security, DHSS, 1989), a similar distinction is apparent. Consumption of soup and chicken, clearly meal items, show the same patterns of regional variation in popularity that the National Food Survey (Ministry of Agriculture, Fisheries and Food, MAFF. 1985) shows among 


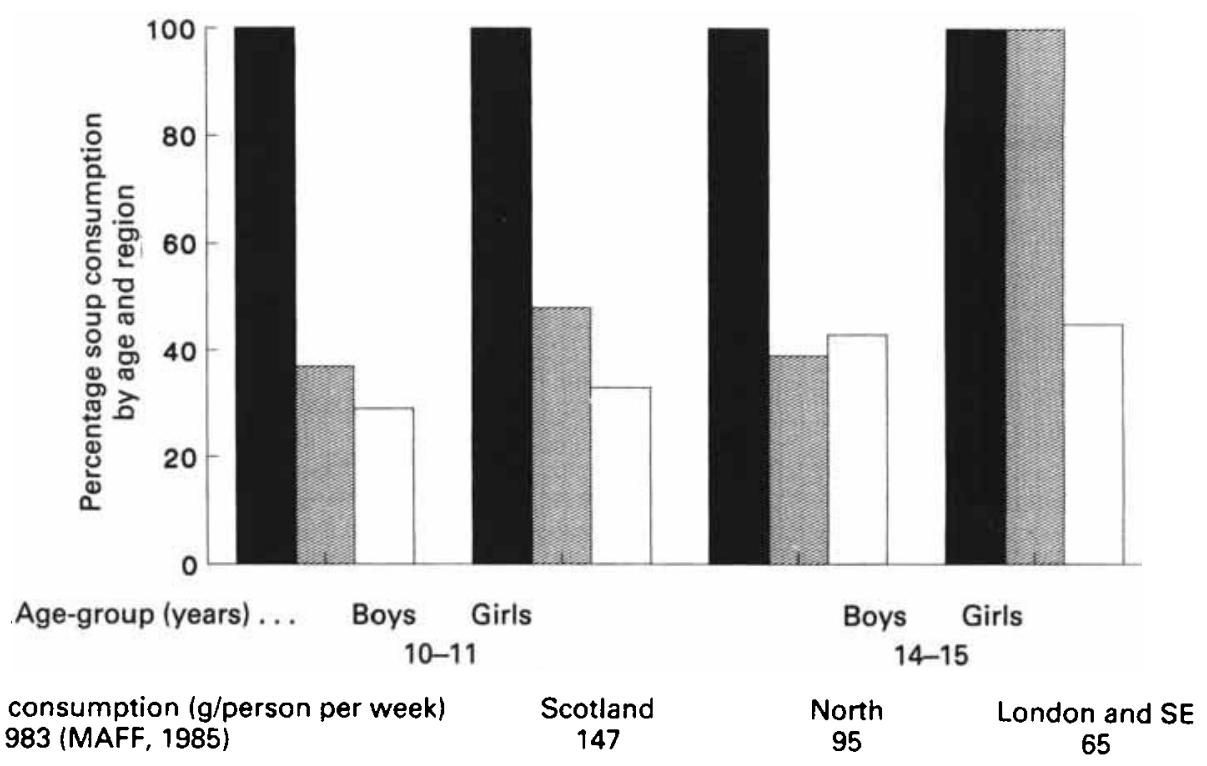

Average consumption ( $g$ /person per week) NFS, 1983 (MAFF, 1985)

Scotland: $\mathbb{Z}$ North; $\square$ London and SE.

Fig. 2. Soup consumption by age and region (DHSS, 1989)

Table 1. Percentage contribution of specific foods to average fat intakes among boys and girls (Department of Health and Social Security, 1989)

\begin{tabular}{|c|c|c|c|c|}
\hline \multirow[t]{2}{*}{ Age group (years) ... } & \multicolumn{2}{|c|}{$10-11$} & \multicolumn{2}{|c|}{$14-15$} \\
\hline & Boys & Girls & Boys & Girls \\
\hline \multicolumn{5}{|l|}{ Percentagc fat from: } \\
\hline Milk & 12 & 12 & 10 & 9 \\
\hline Chips & 8 & 8 & 11 & 11 \\
\hline Other meat products & 7 & 7 & 9 & 8 \\
\hline Biscuits & 8 & 8 & 6 & 6 \\
\hline Carcass meat & 6 & 6 & 7 & 7 \\
\hline Crisps & 6 & 7 & 4 & 6 \\
\hline Butter & 6 & 6 & 7 & 5 \\
\hline Other foods & 47 & 46 & 46 & 48 \\
\hline
\end{tabular}

adults (see Figs. 1 and 2), with greater consistency at younger ages, as might be expected. However, when consumption of 'youth foods' like crisps and fizzy drinks are considered, there is very little difference by region or social class in each age-group.

This dichotomy between adult foods (usually eaten at meals) and peer-oriented perception and use of foods may have important implications for those involved in nutrition education. For example, if one is concerned about moderating fat intake, it is important to know whether the highest proportion of fat intake is coming from foods eaten at meal times, which are still largely under the control of parents, or from 'youth foods' eaten in a different context. Table 1 suggests that it is not the latter which are making the major contribution to fat intakes. Thus, educational attacks on these foods may not only be inappropriate but the strong identification of certain foods with youth 
Table 2. Key features of adolescent eating behaviour

(from Truswell \& Darnton-Hill, 1981)

\begin{tabular}{lll}
\hline Changing meal patterns & Skipping meals \\
& Snacking \\
& Unconventional composition \\
& Fast foods \\
Changing food consumption & Alcohol \\
& Coffec \\
& Likes and dislikes \\
& Appearance \\
\hline
\end{tabular}

culture may also make them very resistant to change and initiate a more generalized rejection of 'nutrition education'. Clearly trying to influence consumption of nutrients that are principally provided by meals may present a need for a very different strategy than would be appropriate if nutrients provided principally by snack foods were of interest.

\section{ADOLESCENTS}

Key features of adolescent eating behaviour have been identified by Truswell \& Darnton-Hill (1981) as indicated in Table 2. Many of these can be associated with the need to express freedom from parental control and adoption of what are perceived to be adult tastes and life-styles.

Although concern about appearance starts much earlier, it is often not until adolescence that children have the freedom to choose foods to the degree that will substantially affect their body shape and size. Wardle \& Beales (1986) found a high level of dissatisfaction with body shape amongst both boys and girls. However, while $52 \%$ of the girls considered that they were overweight and only $10 \%$ too thin, more boys $(34 \%)$ considered themselves too thin and fewer judged themselves to be overweight $(27 \%)$. The extent to which this concern is translated into behaviour is uncertain and the results may be a reflection of the different ways in which the question is asked. In response to the question 'are you dieting?' similar responses were obtained from girls in studies by Bull (1985), and the DHSS (1989), indicating 5-6\% of girls were dieting. In Wardle \& Beales' (1986) study the value was higher, 15\%. In contrast, a study by Thomas (1974) found that while $16 \%$ of girls claimed to be altering their diet in order to lose weight, as many as $24 \%$ said that they were currently trying to lose weight. This higher value included those who were trying to do so by increasing the amount of exercise they took. Increasing exercise was the method generally favoured by boys who wanted to lose weight.

The various influences on the 10-15 years age-group result in great variability within and between sexes in relation to many foods. If we just consider consumption of those foods which are often promoted as being more 'healthy', as indicated in Figs. 3-5, at the age of 10-11 years the only significant differences between the boys and the girls was in frequency of eating salads. However, by the age of 14-15 years girls are also eating citrus fruit and fruit juice as well as salad, more often than boys of the same age. Comparing the different age-groups, there were also significant changes within sexes, with both boys 


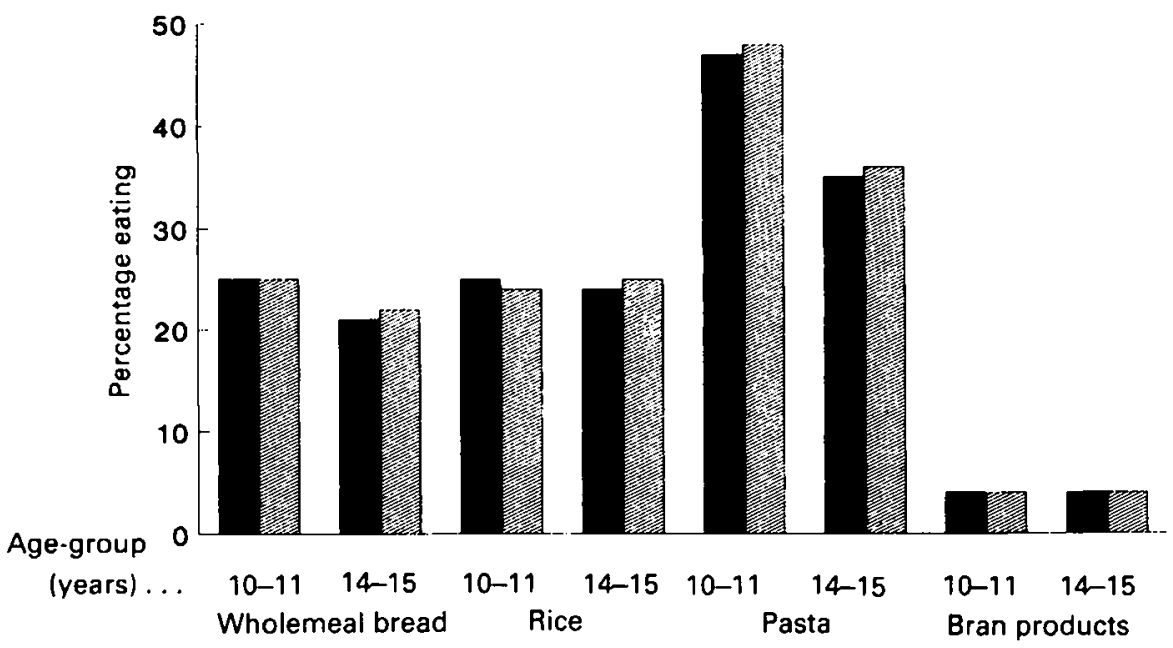

Fig. 3. Consumption of sclected 'healthy' foods by age and sex (DHSS, 1989). Boys; Girls.

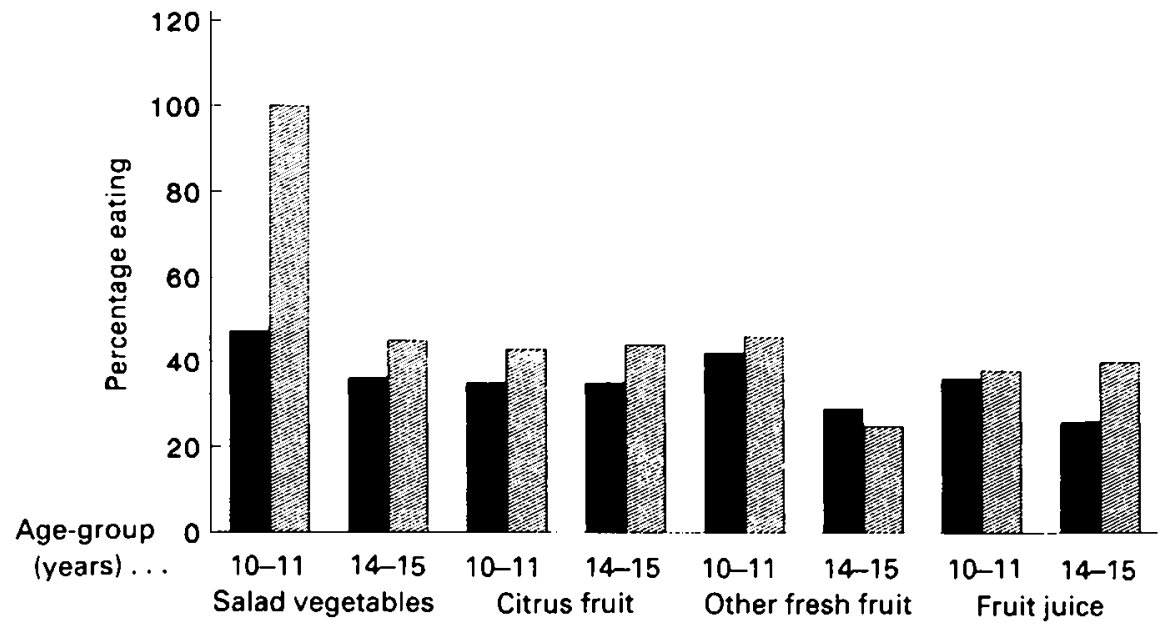

Fig. 4. Consumption of selected 'healthy' foods by age and sex (DHSS, 1989). Boys; Girls.

and girls less likely to eat pasta, salad vegetables and fresh fruit at age 14-15 years. Both boys and girls reported more frequent use of vegetable oil in the older age-group while only boys showed a reduction in eating nuts.

Nutrition education has to operate against a changing background of autonomy over food choices and food ideologies as children grow up. Worsley \& Leitch (1981) have emphasized the differences between the approach of the educator and the student in relation to food. Food preferences and experiences are likely to differ, while concepts of food may differ in content and the importance attached to them.

Inevitably foods are conceptualized within the framework of an individual's 


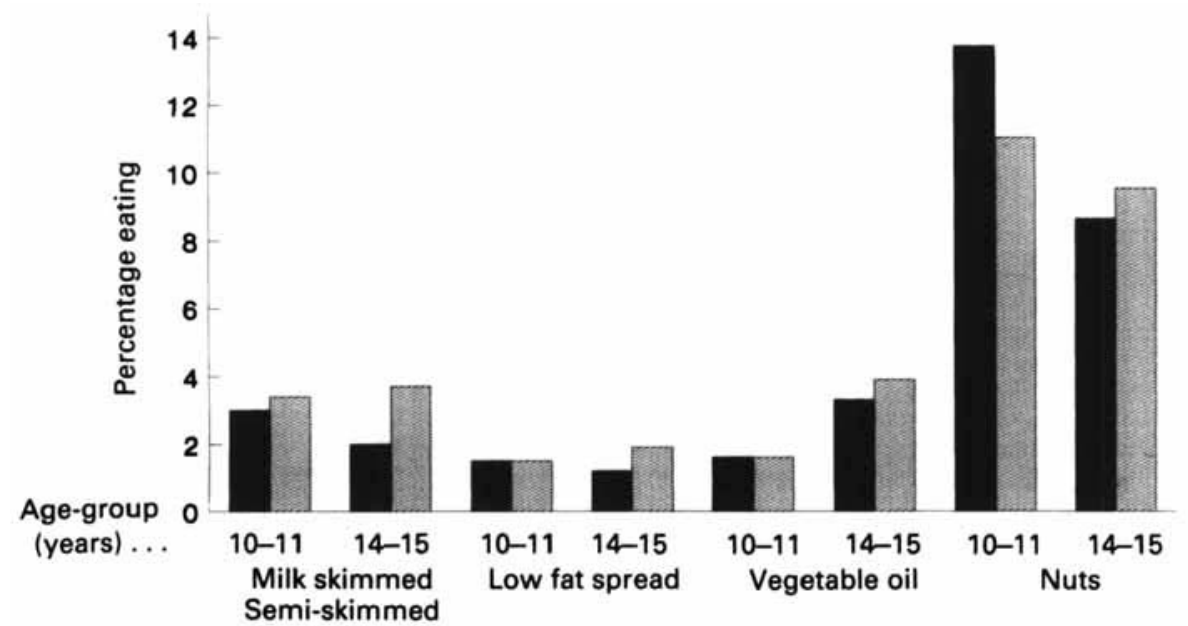

Fig. 5. Consumption of selected 'healthy' foods by age and sex (DHSS, 1989). Boys; $\square$ Girls.

psychosocial goals. If teachers and students do not share each other's life-styles and goals, they are unlikely to eat similar foods or hold similar concepts of food. Unless nutrition education takes account of the eating practices, food concepts and psychosocial goals of the target group it is likely to be seen as an irrelevance.

\section{REFERENCES}

Beauchamp. G. K. \& Moran, M. (1982). Dietary experience and sweet taste preference in human infants. Appetite 3, 139-152.

Birch, L. L. (1979a). Dimensions of pre-school children's food preferences. Journal of Nutrition Education 11, 91-95.

Birch, L. L. (1979b). Pre-school children's preferences and consumption patterns. Journal of Nutrition Education 11, 189-192.

Birch, L. L. (1980). Effects of peer models' food choices and eating behaviours on pre-schoole 's' food preferences. Child Development 51, 489-496.

Birch, L. L. (1981). Generalization of a modified food preference. Child Development 52, 755-758.

Birch, L. L. (1987). The acquisition of food acceptance patterns in children. In Eating Habits. Food, Physiology and Learned Behaviour [R. A. Bookes. D. A. Popplewell and M. J. Burton, editors]. Chichester: Wiley.

Birch, L. L., Zimmerman, S. \& Hind, H. (1980). The influence of social affective context on pre-school children's food preferences. Child Development 51, 856-861.

Bull, N. L. (1985). Dietary habits of 15-25 year olds. Human Nutrition: Applied Nutrition 39A, Suppl.

Casey, R. \& Rozin, P. (1989). Changing children's food preferences: Parent opinions. Appetite 12, 171-182.

Crook, C. K. (1978). Taste perception in the newborn infant. Infant Behaviour and Development 1, 52-69.

Department of Health and Social Security (1989). The Diets of British Schoolchildren. Committee on Medical Aspects of Food Policy, Subcommittee on Nutritional Surveillance. Report on Health and Social Subjects no. 36. London: H.M. Stationery Office.

Desor, J. A., Greene, L. S. \& Maller, O. (1975). Preferences for sweet and salty in 9-15 year old and adult humans. Science 190, 686-687.

Desor, J. A., Maller. O. \& Greene, L. S. (1977). Preference for sweet in humans: Infants, children and adults. In Taste and Development: The genesis of sweet preference, pp. 161-172 [J. M. Weiffenbach, editor]. Bethesda, MD: US Department of Health, Education and Welfare. 
Desor. J. A.. Maller, O. \& Turner, R. E. (1973). Taste in acceptance of sugars by human infants. Journal of Comparative Physiology and Psychology 84, 496-501.

Escalona, S. K. (1945). Feeding disturbances in very young children. American Journal of Orthopsychiatry 15. 76-80.

Fomon, S. J. (1974). Infant Nutrition, 2nd ed. Philadelphia: W. B. Saunders.

Galef. B. G. (1986). Social interaction modifies learned aversions, sodium appetite and both palatability and handling time-induced preferences in rats. Journal of Comparative Psychology 100, 432-439.

James, A. (1981). Confections, concoctions and conceptions. In Popular Culture: Past and Present [B. Waite. editor]. London: Croom Helm.

Jerome, N. W. (1977). Taste experience and the development of a dictary preference for sweet in humans: ethnic and cultural variations in early taste experience. In Taste and Development: The genesis of sweet preference. pp. 235-248 [J. M. Weiffenbach, editor]. Bethesda. MD: US Department of Health. Education and Welfare.

Macarthy, D. (1935). Children's feeding problems in relation to the food aversions in the family. Child Development 6, 277-284.

Ministry of Agriculture, Fisheries and Food (1985). Household food consumption and expenditure. 1983. Annual Report of the National Food Survey Committee. London: H.M. Stationery Office.

Pliner, P. (1983). Family resemblances in food preferences. Journal of Nutrition Education 15, 137-140.

Pliner, P. \& Pelchat, M. L. (1986). Similarities in food preferences between children and their siblings and parents. Appetite 7, 333-342.

Prattala, O. (1988). Sociodemographic difference in fat and sugar consumption patterns among Finnish adolescents. Ecology of Food and Nutrition 22, 53-54.

Ritchey. N. \& Olsen, C. (1983). Relationships between family variables and children's preference for consumption of sweet foods. Ecology of Food and Nutrition 13, 257-266.

Rousseau, N. (1984). Bites and pieces. PhD Thesis, University of Edinburgh.

Rozin, P. \& Schiller. D. (1980). The nature and acquisition of a preference for chilli pepper by humans. Motivation and Emotion 4, 77-101.

Thomas, J. (1974). The nutritional knowledge and practices of young adolescents and their beliefs about overweight. MMedSci Thesis, University of Nottingham.

Truswell, A. S. \& Darton-Hill, I. (1981). Food habits of adolescents. Nutrition Reviews 39. $73-88$.

Wardle. J. \& Beales, S. (1986). Restraint, body image and food attitudes in children aged 12-18 years. Appetite 7, 209-217.

Worsley, A. \& Leitch, D. (1981). Students' perception of favourite and disliked foods. Journal of Human Nutrition 35, 173-187. 